
Solar Corona

C. J. Schrijver et al.

This copy is for your personal, non-commercial use only.

If you wish to distribute this article to others, you can order high-quality copies for your colleagues, clients, or customers by clicking here.

Permission to republish or repurpose articles or portions of articles can be obtained by following the guidelines here.

The following resources related to this article are available online at www.sciencemag.org (this information is current as of May 6, 2013):

Updated information and services, including high-resolution figures, can be found in the online version of this article at:

http://www.sciencemag.org/content/335/6066/324.full.html

Supporting Online Material can be found at:

http://www.sciencemag.org/content/suppl/2012/01/18/335.6066.324.DC1.html

http://www.sciencemag.org/content/suppl/2012/01/19/335.6066.324.DC2.html

A list of selected additional articles on the Science Web sites related to this article can be found at:

http://www.sciencemag.org/content/335/6066/324.full.html\#related

This article cites 11 articles, 2 of which can be accessed free:

http://www.sciencemag.org/content/335/6066/324.full.html\#ref-list-1

This article has been cited by 1 articles hosted by HighWire Press; see:

http://www.sciencemag.org/content/335/6066/324.full.html\#related-urls

This article appears in the following subject collections:

Planetary Science

http://www.sciencemag.org/cgi/collection/planet_sci 
25. Materials, methods, and additional control data are available as supporting material on Science Online

26. K. L. Heredia et al., J. Am. Chem. Soc. 127, 16955 (2005).

27. M. Matsumura, B. W. Matthews, Science 243, 792 (1989).

28. S. Rosenblatt et al., Nano Lett. 2, 869 (2002).

29. R. ]. Chen et al., Proc. Natl. Acad. Sci. U.S.A. 100, 4984 (2003).

30. R. J. Chen et al., J. Am. Chem. Soc. 126, 1563 (2004).

31. S. O. Meroueh et al., Proc. Natl. Acad. Sci. U.S.A. 103 4404 (2006).

32. J. Kong et al., Science 287, 622 (2000).

33. P. G. Collins, K. Bradley, M. Ishigami, A. Zettl, Science 287, 1801 (2000).

34. T. Zhang, S. Mubeen, N. V. Myung, M. A. Deshusses, Nanotechnology 19, 332001 (2008).

35. A. Tsugita, M. Inouye, E. Terzaghi, G. Streisin, J. Biol. Chem. 243, 391 (1968)

36. H. B. Jensen, K. Kleppe, Eur. J. Biochem. 28, 116 (1972).

37. Y. Chen, D. Hu, E. R. Vorpagel, H. P. Lu, J. Phys. Chem. B 107, 7947 (2003)

38. S. N. Xie, Single Molecules 2, 229 (2001)
39. H. P. Lu, L. Y. Xun, X. S. Xie, Science 282, 1877 (1998)

40. D. Hu, H. P. Lu, Biophys. J. 87, 656 (2004)

41. H. P. Lu, Curr. Pharm. Biotechnol. 5, 261 (2004).

42. Y. Wang, H. P. Lu, J. Phys. Chem. B 114, 6669 (2010).

43. G. E. Arnold, ]. I. Manchester, B. D. Townsend, R. L. Ornstein, J. Biomol. Struct. Dyn. 12, 457 (1994).

44. B. L. de Groot, S. Hayward, D. M. F. van Aalten, A. Amadei, H. J. C. Berendsen, Proteins 31, 116 (1998).

45. H. R. Faber, B. W. Matthews, Nature 348, 263 (1990).

46. K. Svoboda, P. P. Mitra, S. M. Block, Proc. Natl. Acad. Sci. U.S.A. 91, 11782 (1994).

47. M. ]. Schnitzer, S. M. Block, Cold Spring Harb. Symp. Quant. Biol. 60, 793 (1995).

48. W. L. Xu, ]. S. Kong, P. Chen, J. Phys. Chem. C 113, 2393 (2009).

49. M. J. Schnitzer, S. M. Block, Nature 388, 386 (1997).

50. G. Bhabha et al. Science 332, 234 (2011).

51. H. S. Mchaourab, K. J. Oh, C. J. Fang, W. L. Hubbell, Biochemistry 36, 307 (1997)

52. E. Stern et al., Nano Lett. 7, 3405 (2007).

53. I. Heller et al., Nano Lett. 8, 591 (2008).
54. L. Prisbrey, G. Schneider, E. Minot, J. Phys. Chem. B 114, 3330 (2010)

55. C. Li et al., J. Am. Chem. Soc. 127, 12484 (2005).

56. S. R. Hunt, D. Wan, V. R. Khalap, B. L. Corso, P. G. Collins, Nano Lett. 11, 1055 (2011).

57. B. Brooks, M. Karplus, Proc. Natl. Acad. Sci. U.S.A. 82, 4995 (1985).

Acknowledgments: This work was sponsored by the National Cancer Institute of the NIH (R01 CA133592-01 and T32CA009054) and NSF (DMR-0801271, ECCS-0802077, and the Center for Chemical Innovation on Chemistry at the Space-Time Limit CHE-0802913).

\section{Supporting Online Material}

www.sciencemag.org/cgi/content/full/335/6066/319/DC1

Materials and Methods

SOM Text

Figs. $\mathrm{S} 1$ to $\mathrm{S} 6$

Tables S1 and S2

References (58-74)

4 October 2011; accepted 5 December 2011 10.1126/science. 1214824

\title{
Destruction of Sun-Grazing Comet C/2011 N3 (SOHO) Within the Low Solar Corona
}

\author{
C. J. Schrijver, ${ }^{1 *}$ J. C. Brown, ${ }^{2}$ K. Battams, ${ }^{3}$ P. Saint-Hilaire, ${ }^{4}$ W. Liu, ${ }^{1,5}$ \\ H. Hudson, ${ }^{2,4}$ W. D. Pesnell ${ }^{6}$
}

Observations of comets in Sun-grazing orbits that survive solar insolation long enough to penetrate into the Sun's inner corona provide information on the solar atmosphere and magnetic field as well as on the makeup of the comet. On 6 July 2011, the Solar Dynamics Observatory (SDO) observed the demise of comet $\mathrm{C} / 2011 \mathrm{N3}$ (SOHO) within the low solar corona in five wavelength bands in the extreme ultraviolet (EUV). The comet penetrated to within 0.146 solar radius ( 100,000 kilometers) of the solar surface before its EUV signal disappeared. Before that, material released into the coma-at first seen in absorption-formed a variable EUV-bright tail. During the final 10 minutes of observation by SDO's Atmospheric Imaging Assembly, $\sim 6 \times 10^{8}$ to $6 \times 10^{10}$ grams of total mass was lost (corresponding to an effective nucleus diameter of $\sim 10$ to 50 meters), as estimated from the tail's deceleration due to interaction with the surrounding coronal material; the EUV absorption by the comet and the brightness of the tail suggest that the mass was at the high end of this range. These observations provide evidence that the nucleus had broken up into a family of fragments, resulting in accelerated sublimation in the Sun's intense radiation field.

$\mathrm{D}$ uring its 15 years of operation, the Large Angle and Spectrometric Coronagraph (LASCO) (1) onboard the Solar and Heliospheric Observatory (SOHO) has observed more than 2000 comets as they approached the

${ }^{1}$ Lockheed Martin Advanced Technology Center, 3251 Hanover Street, Palo Alto, CA 94304, USA. ${ }^{2}$ School of Physics and Astronomy, University of Glasgow, Glasgow G12 8QQ, UK. ${ }^{3} \mathrm{Naval}$ Research Laboratory, Code 7663, 4555 Overlook Avenue, SW Washington, DC 20375, USA. ${ }^{4}$ Space Sciences Laboratory, University of California, Berkeley, CA 94720, USA. ${ }^{5}$ W. W. Hansen Experimental Physics Laboratory, Stanford University, Stanford, CA 94305, USA. ${ }^{6}$ Code 671, NASA Goddard Space Flight Center, Greenbelt, MD 20771, USA.

*To whom correspondence should be addressed. E-mail: schrijver@lmsal.com
Sun. The population of Sun-grazing comets is dominated by the Kreutz group, which orbit to within one to two solar radii from the solar surface with orbital periods of 500 to 1000 years. More than 1400 of the comets seen by SOHO are members of this group, making it the largest known group of comets, likely originating from the breakup of a progenitor body as recently as 2500 years ago (2). Only the largest of the Kreutz-group comets [with diameters up to $\sim 100 \mathrm{~m}$ (2)] have survived closest approach (perihelion), and SOHO never witnessed such a survival (3) until sungrazing comet Lovejoy emerged after its perihelion passage on the Sun's far side on 15-16 December 2011. Most of the destructions occurred well before perihelion, but some occurred after the comet disappeared behind the occulting disk of the coronagraph. None could be followed into the Sun's lower atmosphere.

Here, we report on the observed destruction of comet $\mathrm{C} / 2011 \mathrm{~N} 3$ (SOHO) within the solar atmosphere. We use EUV images obtained with the Atmospheric Imaging Assembly [AIA (4)] on the Solar Dynamics Observatory (SDO), which show different parts of the comet in absorption and in emission against the background EUV emission from the hot $\left(1 \times 10^{6}\right.$ to $\left.3 \times 10^{6} \mathrm{~K}\right)$ outer solar atmosphere (the corona). AIA images the entire visible hemisphere of the Sun, including its off-disk corona, at 12-s intervals for sets of eight distinct (E)UV channels, at a resolution of $\sim 1.2$ arc sec, and with a high signalto-noise ratio achieved with exposures no longer than $2.3 \mathrm{~s}$. The comet's speed of $\sim 600 \mathrm{~km} / \mathrm{s}$ caused blurring in the AIA exposures that is only slightly in excess of the instrumental resolution. This detailed view of the solar corona enabled us to track the comet's tail and to determine the comet's orbit across much of the solar disk until it faded within $20 \mathrm{~min}$ of its first appearance. From Fig. 1 and movies S1 and S2, it appears that the comet's nucleus had fragmented.

The comet $\mathrm{C} / 2011 \mathrm{~N} 3$ (SOHO) was first seen in AIA's $171 \AA$ EUV channel while it was $\sim 0.2$ solar radii in projection off the solar limb, roughly at 5 July 2011 23:46 UTC (all times are given in UTC for photon arrival times at SDO, in geosynchronous orbit). The comet could be tracked in AIA images until approximately 6 July 2011 00:05:50, when it faded from all five EUV channels in which it was visible $(131,171$, 193, 211, and $335 \AA$ ). Observations made by the Extreme-Ultraviolet Imager (EUVI) on the SolarTerrestrial Relations Observatory [STEREO (5)] B show a faint signal of the comet high above the solar surface from its near-quadrature view relative to the Sun-Earth line (and thus against a background coronal emission weaker than from AIA's perspective by nearly a factor of 10) up to 
Fig. 1. (A) EUV image of the solar corona (in AIA's $171 \AA$ channel, most sensitive to coronal emission near $10^{6} \mathrm{~K}$ ) at 6 July 2011 00:00:01 UTC. Overlaid (in black) is the projected orbit of the comet C/2011 N3 between 5 July 2011 23:46 UTC and 6 July 2011 00:06 UTC. Orbital positions marked by plus signs were used as the threedimensional starting points to trace the Sun's magnetic field through a potential field source surface (PFSS) approxi-
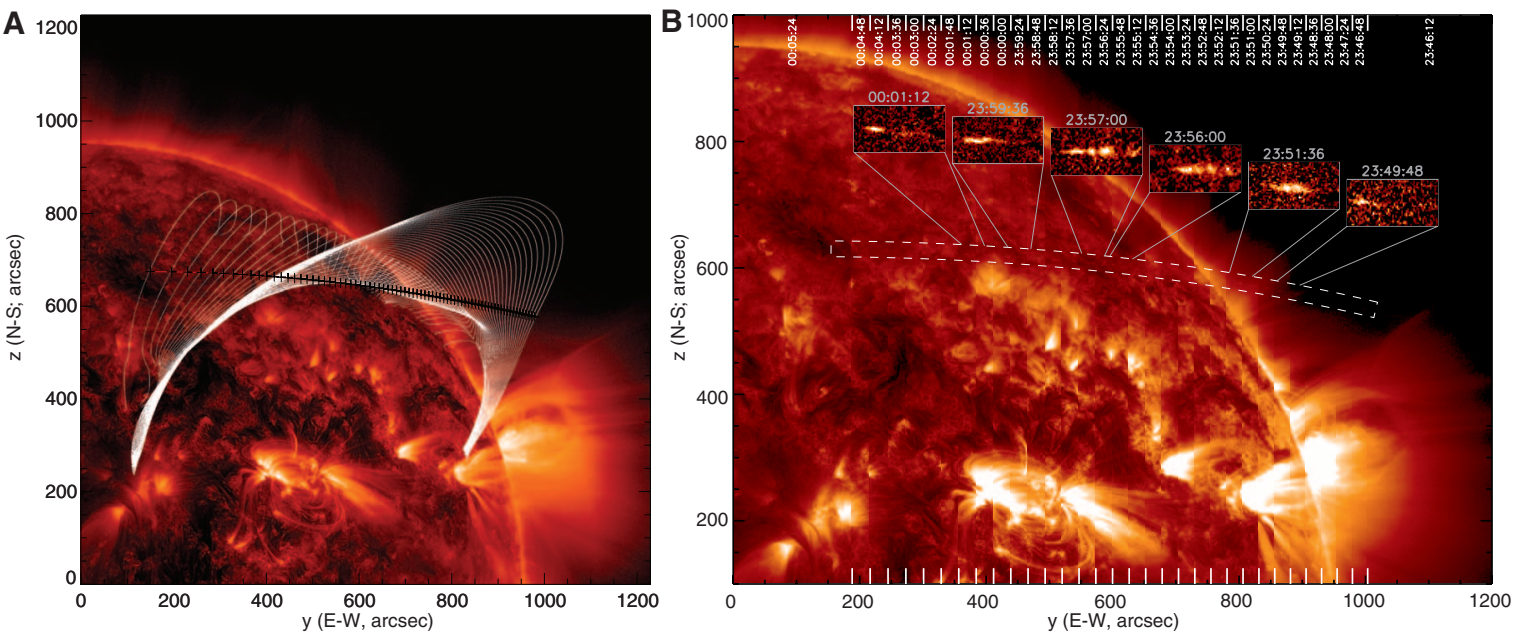

mation (9); all of the traced fields lead to the solar surface in both directions, showing that the comet in this time interval moved through the closed-field corona rather than though the solar wind. (B) Composite AIA $171 \AA$ A images in which vertical strips (marked on the axes, with UTC time stamps) are concatenated as the comet (moving within the dashed outline) is within that strip (see movie S1 for a sequence of unaltered images). Insets (with UTC times) show intensity differences for images containing the comet and images taken $36 \mathrm{~s}$ earlier, thus removing the background corona. Note that the EUV-bright tail does not align with the field lines. In both panels, $1 \mathrm{arc} \mathrm{sec} \approx 740 \mathrm{~km}$.
$00: 11: 28$ UTC, but the comet is not visible in the next image at 00:12:43 UTC. The AIA observations during this later phase of STEREO observations have intensity upper limits at least an order of magnitude lower than the average intensity during AIA visibility (see below), so that the comet's mass loss in that late phase observed by STEREO was probably much smaller than when it was visible to AIA.

The trajectory of the comet (Fig. 2) determined from our observations agrees well with the orbit determined from observations with the SOHO/LASCO and STEREO/SECCHI (Sun Earth Connection Coronal and Heliospheric Investigation) (5) coronagraphs. The match of the projected orbit is better than $\sim 3$ arc sec and $\sim 15 \mathrm{~s}$, implying that there was no significant deceleration of the nuclear body or major fragments within the solar corona (consistent with the estimate of the cross section) $(6,7)$. This close match gives us confidence that we know the height of the comet's trajectory above the solar surface to within a few thousand kilometers. The orbit of the comet (8), determined by the IAU Minor Planet Center, shows an orbital perihelion time of 6 July 20116.00232 TT (corresponding to a photon-arrival time at SDO of 6 July 2011 00:11:11 UTC) and an orbital perihelion parameter of $q=0.0052986$ (corresponding to a height above the solar surface of $97,200 \mathrm{~km}$ ).

After manually tracing the comet's bright tail (extending over $\sim 10^{4} \mathrm{~km}$ ) in the AIA images, we slid a box along a parabolic fit to the traced positions to determine the intensity variations during the comet's final phase as it moved through the solar corona (Fig. 3). The light curves in all but one of the coronal emission lines (i.e., excluding the low-signal $94 \AA$ channel as well as the $\mathrm{He}^{+} 304 \AA$ channel with a high background from the low solar atmosphere) show that the total signal varied on a 100 -s time scale by up to a factor of $\sim 4$. The various wavelengths show
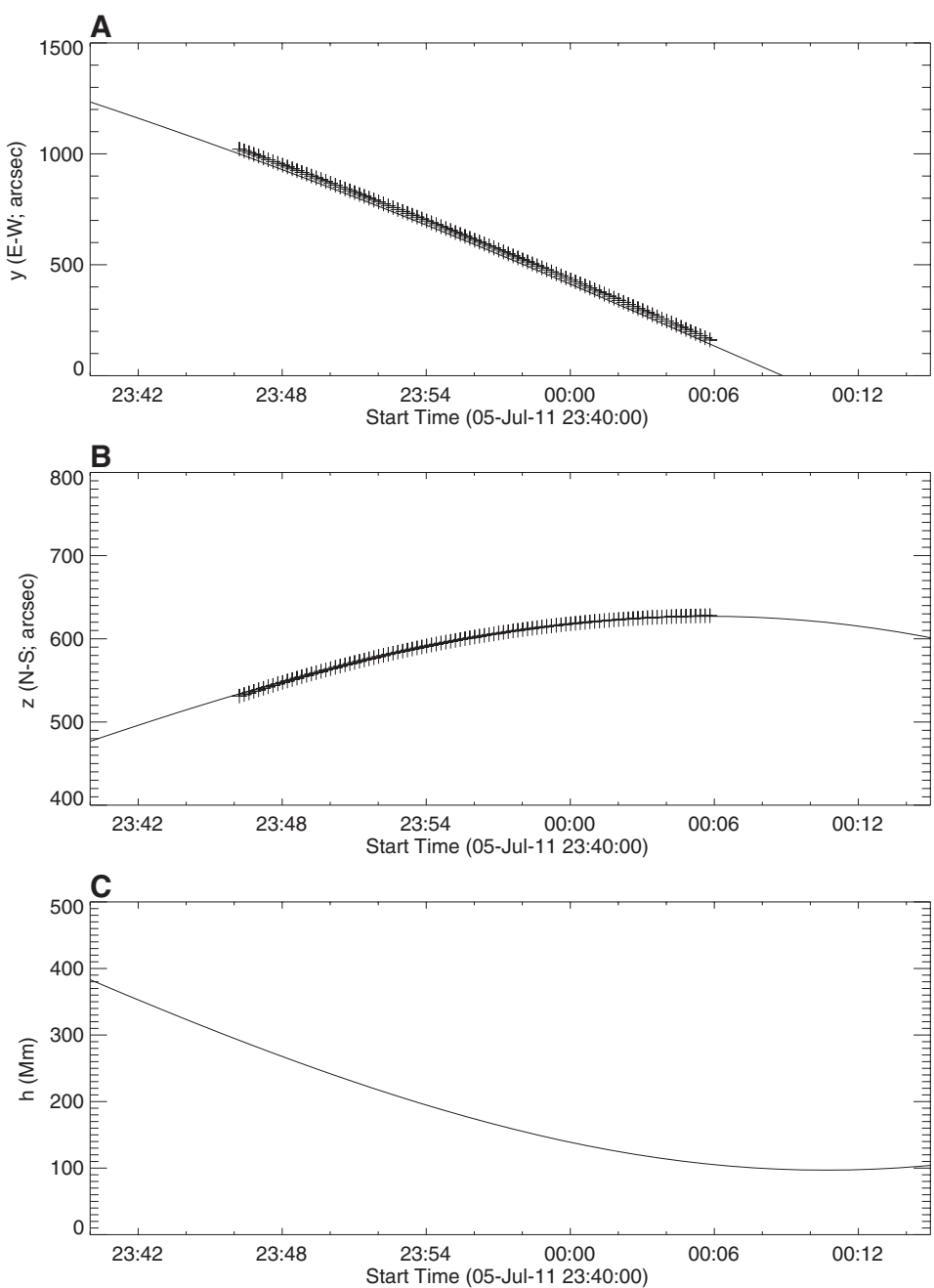

Fig. 2. (A to C) Orbital details of the comet nucleus derived from the orbital parameters for the comet from the Minor Planet Center (16), converted to photon-arrival times at Earth in UTC [(A) and (B)] and the corresponding height above the solar surface $(C)$. The $y$ coordinate is positive to solar west and the $z$ coordinate positive to solar north; 1 arc sec $\approx 740 \mathrm{~km}$. The plus signs show the tracked positions in the SDO/AIA images (Fig. 1) interpolated from a smooth parabolic fit to the original measurements. 
near-synchronous changes, implying that this variability was real. Variations in the solar magnetic field at the height of the comet above the surface are expected to be small. A model potential magnetic field (9) (Fig. 1A) shows closed fields that increase in field strength from 0.4 to $0.8 \mathrm{G}$ along the trajectory marked in the figure. The observed variability likely reflects varying rates of sublimation of the comet nucleus, possibly owing to a combination of rotation, heterogeneous nuclear structure, and sequential fragmentation events; we see evidence in the images of several fragments
(Fig. 1B) and have indirect evidence for multiple fragments (7). The positive and negative intensity variations in the He II $304 \AA$ channel partly reflect the high background emission, resulting in a lower signal-to-noise ratio, possibly compounded by opacity effects in the cometary tail.
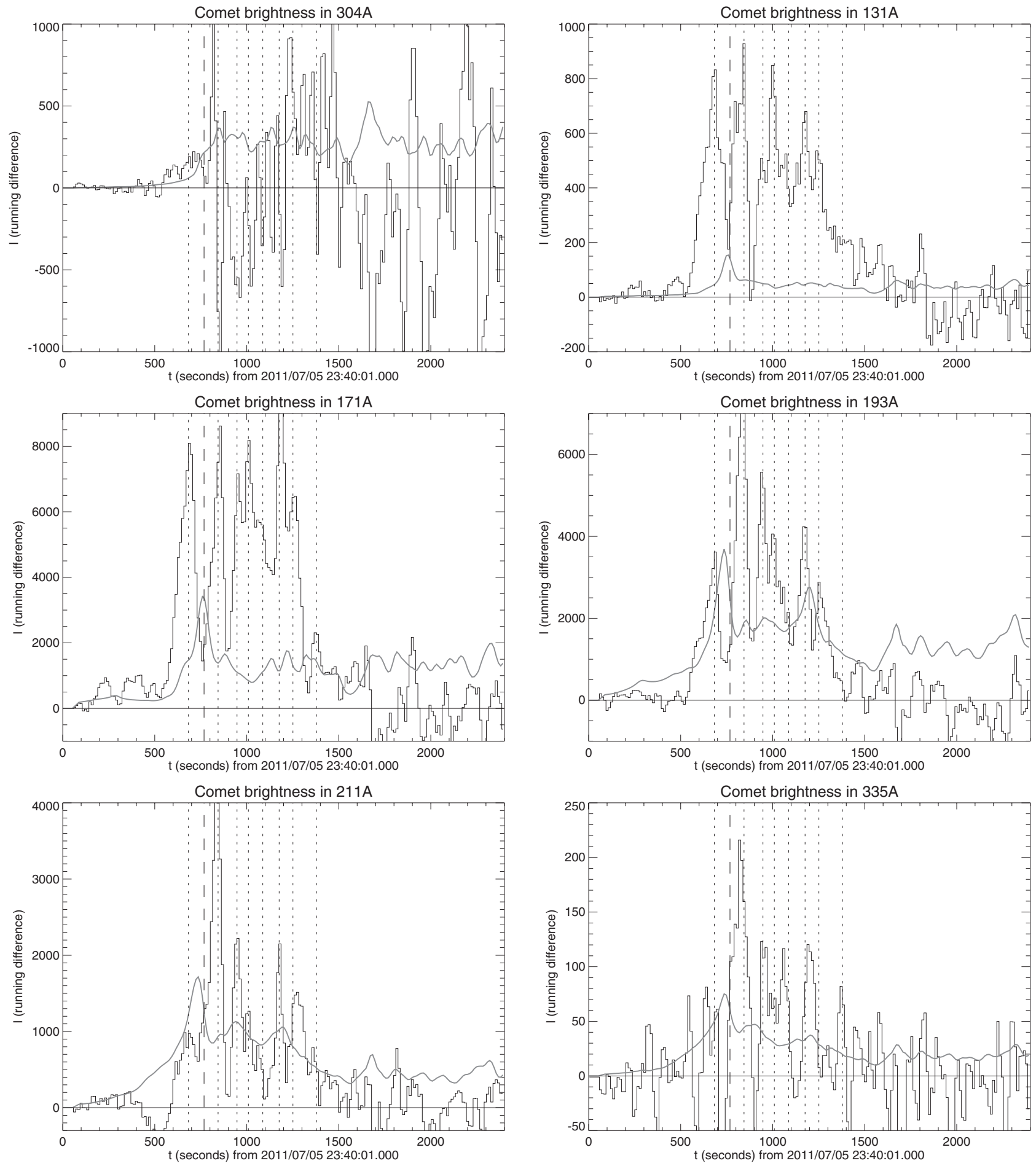

Fig. 3. Brightness I as a function of time for six EUV channels of SDO/AIA for the comet $(304,131,171,193,211$, and $335 \AA$ ) after subtraction of the coronal background brightness $I_{\mathrm{b}}$ in the same locations $36 \mathrm{~s}$ earlier. The brightness is measured by summing over a box 30 pixels wide by 15 pixels high (or $13,300 \mathrm{~km}$ by $6700 \mathrm{~km}$ ) that moves with the comet,

starting behind the absorption feature of the inner, dense part of the coma (at $x=0$ in Fig. 4). Dotted lines mark times of peak comet brightness in $171 \AA$; dashed lines mark the edge of the Sun. For comparison, the scaled coronal background brightness, $I_{b} / 100$, is shown by the gray curves. 
The AIA observations show an absorption signature in a region just ahead of the tail's EUV emission. This feature, seen most strongly when the comet crossed the solar limb, reveals the densest inner part of the comet's coma. A series of space-time diagrams (Fig. 4) (7) show that this region was typically 7 arc $\sec (\sim 5000 \mathrm{~km})$ ahead of the EUV-bright tail. The diameter of the coma's absorption feature as seen in the $193 \AA$ channel at the half-intensity point is $d_{193} \approx 1700 \pm$ $300 \mathrm{~km}$, which exceeds the resolution of $\ell_{193}=$ $1000 \mathrm{~km}$ for the $193 \AA$ AIA channel derived from ray-tracing modeling (10); the observed radius of the coma's dense inner region can thus be estimated from the combined effect of intrinsic width and instrumental resolution through $d_{193}^{2}-\ell_{193}^{2}$ to be $1400 \pm 600 \mathrm{~km}$. This value is comparable to the motion blurring during the 2.3-s exposure, so it constitutes an upper limit. If the coma were completely opaque, the intensity contrast of $\sim 1 \%$ against the background corona (with most of the corona behind it) would require a radius of $\sim 50 \mathrm{~km}$; hence, we can set a range for the coma's radius $r_{\text {coma }}=50$ to $700 \mathrm{~km}$.

During the $\sim 600 \mathrm{~s}$ when the tail was brightest in the EUV, the time-distance diagram (Fig. 4) shows a series of arches behind the nucleus. We interpret these as tracks of variable amounts of released material decelerating behind the nucleus as that material interacted with the hot $\left(\sim 10^{6} \mathrm{~K}\right)$ coronal plasma, with relative kinetic energy of $2 \mathrm{keV}$ per nucleon. The inertia of the sublimated and subsequently ionized material must therefore have sufficed, at least within much of the EUVbright tail, to largely outweigh the Lorentz force induced by motion through the coronal field (distorting the magnetic field, rather than allowing that field to determine the plasma motion). The decelerated tail was deflected only weakly to the north as the comet traveled across the solar disk (7).

We were able to measure the deceleration of the material lost from the nucleus only because the sublimation rate was highly variable, producing discrete episodes of enhanced mass loss. The variability in the tail's brightness was not caused by density contrasts in the background corona; the coronal emission just before the time when the nucleus passed over a given location exhibited much smaller variations. Because we know the comet's orbital trajectory and speed, know the time scale for the deceleration of the tail, and have an approximate knowledge of the plasma density in the quiet-Sun corona (11) along that trajectory, we can estimate the mass loss rate and total mass lost during the visibility in the AIA images (7): $10^{6}$ to $10^{8} \mathrm{~g} / \mathrm{s}$ and $6 \times 10^{8}$ to $6 \times 10^{10} \mathrm{~g}$, respectively.

The mass loss rate estimated from the tail deceleration can be compared to the mass lost by insolation during the same period (12) on the basis of the relationship between heat input and the latent heat of vaporization of cometary materials, the specific heat of sublimation, and the mean mass density of the nucleus (7). This comparison suggests that the comet's nuclear material was contained in a number of fragments, because a single nuclear body would have had too small a cross section to capture the insolation energy needed to sublimate the total mass in the available time. About one to two dozen of these fragments must have been larger than $10 \mathrm{~m}$ in diameter. We see direct observational evidence for fragmentation in the AIA observations (7).

Deexcitation after ionizing collisions should be the main mechanism by which the tail of $\mathrm{C} / 2011 \mathrm{~N} 3$ becomes visible in the EUV. A comet moving at $\sim 600 \mathrm{~km} / \mathrm{s}$ through the solar corona releases grains, molecules, and atoms with energies of $2 \mathrm{keV}$ per nucleon in the coronal rest frame. The deceleration of these particles through collisions with the coronal plasma excites and
Fig. 4. Intensity profiles along eastwest cuts (9 AIA pixels or $3600 \mathrm{~km}$ high) comoving with the comet, stacked into a grayscale display of time versus distance ( 1 arc sec $\approx$ $740 \mathrm{~km}$, with arbitrary offset). AIA channel identifications (mean wavelengths, in angstroms) are shown at the top. The 131, 171, 193, and $211 \AA$ channels exhibit bright arcs that reveal the deceleration of the material in the comet's tail behind the nucleus. A very faint arc in the 171 A channels at $t \approx 400 \mathrm{~s}$ (black arrow) suggests that this process already occurs there (at $h \approx 300,000$ $\mathrm{km}$; Fig. 2) but is much more visible later on, closer to the insolating solar surface.

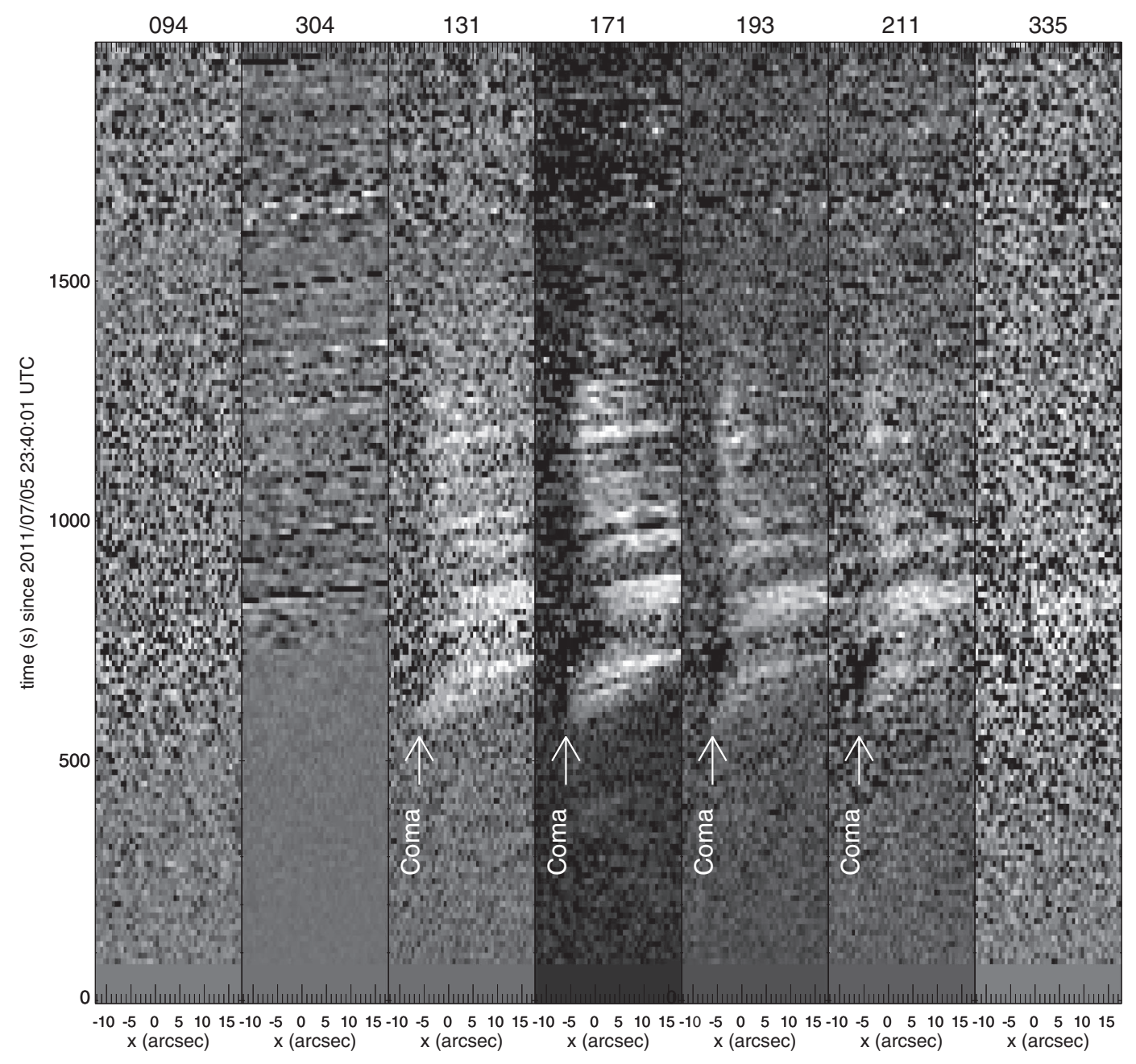


ionizes the cometary material, both by direct collisional excitation and by charge-exchange collisions. For comets far from the Sun, charge-exchange collisions of the cometary material with the solar wind flowing by at relative speeds of $\sim 300$ to $800 \mathrm{~km} / \mathrm{s}$ excite electrons into highly excited states of solar-wind minor ions that subsequently emit EUV or even $\mathrm{x}$-ray photons as they transition to lower-energy states $(13,14)$.

However, one possible explanation for the EUV-bright tail of $\mathrm{C} / 2011 \mathrm{~N} 3$, consistent with our mass estimate from the tail's time-distance behavior (7), is that the ionization and excitation states of the material lost from the nuclear bodies are equilibrated with those of the surrounding coronal plasma. One important aspect in estimating the tail's EUV brightness in the AIA observations is that the comet released a mix of elements very rich in heavy ions relative to the mixture of the solar plasma, which is strongly dominated by hydrogen and helium. Efficient heat conduction into this metal-rich mixture could thus explain why the contrast of tail to coronal brightness is comparable in all of the coronal AIA channels (see Fig. 3). Other emission mechanisms, including collisional ionization (direct or charge-exchange) and emis- sion from elements other than iron, might also be important; we merely argue that at least one mechanism is consistent with the observed tail brightness for the mass estimate based on the observed tail deceleration, provided it lies near the high end of that range (7).

Prior to these observations, cometary masses were generally derived from light curves during their orbits, assumed albedos, and estimated mass densities, or by direct imaging for the few comets that have been visited by spacecraft. $\mathrm{SOHO} / \mathrm{LASCO}$ observes a Sun-grazing comet roughly once every 3 days, and although most fade well before perihelion, several per year should reach the solar corona and be available for further study of cometary properties, as well as probes of the solar corona.

\section{References and Notes}

1. G. E. Brueckner et al., Sol. Phys. 162, 357 (1995).

2. M. M. Knight et al., Astron. J. 139, 926 (2010).

3. M. Iseli, M. Küppers, W. Benz, P. Bochsler, Icarus 155, 350 (2002)

4. J. R. Lemen et al., Sol. Phys. 10.1007/s11207-011-9776-8 (2011).

5. R. A. Howard et al., Space Sci. Rev. 136, 67 (2008).

6. ]. C. Brown, H. E. Potts, L. ]. Porter, G. le Chat, http://arxiv.org/abs/1107.1857 (2011).

7. See supporting material on Science Online.
8. www.minorplanetcenter.net/mpec/K11/K11N41.html; MPEC 2011-N41: COMET C/2011 N3 (SOHO).

9. C. J. Schrijver, M. L. DeRosa, Sol. Phys. 212, 165 (2003)

10. P. Boerner et al., Sol. Phys. 10.1007/s11207-011-9804-8 (2011).

11. M. Guhathakurta, R. R. Fisher, R. C. Altrock, Astrophys. J. 414, L145 (1993).

12. At heights above 0.01 solar radii, collisional heating and ablative mass loss by atmospheric impacts are negligible (6)

13. T. E. Cravens, Science 296, 1042 (2002)

14. C. M. Lisse, T. E. Cravens, K. Dennerl, in Comets II, M. C. Festou, H. U. Keller, H. A. Weaver, Eds. (Univ. of Arizona Press, Tucson, AZ, 2004), pp. 631-643.

Acknowledgments: Supported by NASA SDO/AIA contract NNG04EA00C to Lockheed Martin's Solar and Astrophysics Laboratory. The SDO/AIA data can be accessed at

http://aia.Imsal.com; the STEREO/SECCHI data can be accessed at http://stereo-ssc.nascom.nasa.gov/data/ins data/secchi. We thank the four reviewers and the editor for their help in improving the manuscript.

\section{Supporting Online Material}

www.sciencemag.org/cgi/content/full/335/6066/324/DC1 Materials and Methods

Fig. S1

References (15-21)

Movies S1 and S2

25 July 2011; accepted 22 November 2011 $10.1126 /$ science 121168

\title{
Polymerase Exchange During Okazaki Fragment Synthesis Observed in Living Cells
}

\author{
Giuseppe Lia, ${ }^{1,2,3 *}$ Bénédicte Michel, ${ }^{1,2}$ Jean-François Allemand ${ }^{3,4,5 *}$
}

DNA replication machineries have been studied extensively, but the kinetics of action of their components remains largely unknown. We report a study of DNA synthesis during replication in living Escherichia coli cells. Using single-molecule microscopy, we observed repetitive fluorescence bursts of single polymerase IIIs (Pol IIIs), indicating polymerase exchange at the replication fork. Fluctuations in the amount of DNA-bound single-stranded DNA-binding protein (SSB) reflect different speeds for the leading- and lagging-strand DNA polymerases. Coincidence analyses of Pol III and SSB fluctuations show that they correspond to the lagging-strand synthesis and suggest the use of a new Pol III for each Okazaki fragment. Based on exchanges involving two Pol IIIs, we propose that the third polymerase in the replisome is involved in lagging-strand synthesis.

$\mathrm{D}$ NA replication is carried out in all organisms by a multiprotein complex called the replisome. Owing to the high level of functional similarity of replication proteins in

\footnotetext{
${ }^{1}$ CNRS, Centre de Génétique Moléculaire, UPR3404, Gif-surYvette F-91198, France. '2Université Paris-Sud, Orsay F-91405, France. ${ }^{3}$ Laboratoire de Physique Statistique, Ecole Normale Supérieure (ENS), UMR 8550 CNRS, Universités Pierre et Marie Curie and Paris Diderot, Département de Physique, 24 rue Lhomond, 75231 Paris Cedex 05, France. ${ }^{4}$ Département de Biologie, Institut de Biologie de l'Ecole Normale Supérieure, UMR 8542 CNRS, 46 rue d'Ulm, 75231 Paris Cedex 05, France. ${ }^{5}$ Institut Universitaire de France (IUF), 103, bd Saint-Michel 75005 Paris, France.
}

*To whom correspondence should be addressed. E-mail: allemand@lps.ens.fr (].-F.A.); lia@cgm.cnrs-gif.fr (G.L.) different species from bacteria and phages to eukaryotes, bacterial replication is used as a model system. In Escherichia coli, the replisome consists of 13 proteins that participate in the synchronized DNA synthesis of the leading strand (synthesized continuously) and the lagging strand, made discontinuously as $\sim 1$ - to 2-kb Okazaki fragments (OF) (1-10). The DNA polymerase III holoenzyme (hPol III) is composed of a clamp loader that interacts with three copies of the core polymerase Pol III, two of which act on the two DNA strands ( $\left(\mathrm{Pol}_{\text {lead }}\right.$ and $\left.\mathrm{Pol}_{\text {lag }}\right)(11,12)$. Pol III contains a catalytic subunit, DnaE, and a proofreading subunit, DnaQ. Active Pol IIIs are stabilized on DNA by interactions with the sliding clamp. The DNA helicase DnaB unwinds the template DNA by progressing on the laggingstrand template; it interacts with the hPol III through the clamp loader subunit $\tau$ and with a primase that synthesizes RNA primers for each OF. Single-stranded (ss) DNA-binding proteins (SSB) bind the exposed ssDNA on the laggingstrand template.

A single fluorescent protein can be detected in living $E$. coli cells when its diffusion is reduced (13-15). The replication fluorescent proteins will diffuse slowly enough to be detected only when bound to the replisome. We implemented a version of the detection by localization technique (13-15) with stroboscopic illumination. This limited illumination duration reduces photobleaching, allowing us to probe the dynamics of Pol III in vivo for long periods. We used a dual view system to measure the simultaneous binding times of two differentially labeled replisome components. We expressed fluorescent fusions of the DNA polymerase DnaE, the proofreading subunit DnaQ, and the SSB protein, labeled with either a yellow [enhanced yellow fluorescent protein (eYFP) or yellow fluorescent protein for energy transfer (Ypet)] or red fluorescent protein (mCherry). dnaQ-Ypet and $d n a E$-eYFP were fully functional under our experimental conditions (16) [supporting online material (SOM)]. ssb-mCherry and Ypet fusions were functional only in the presence of the wild-type (wt) $s s b$ gene, but 20 to $50 \%$ of SSB proteins could be labeled, enough to ensure the labeling of most tetramers without causing any defect in cell growth (figs. S1 and S3 and tables $\mathrm{S} 1$ and S2).

As expected, measures of the relative position of DnaQ-YPet and DnaE-eYFP in growing 